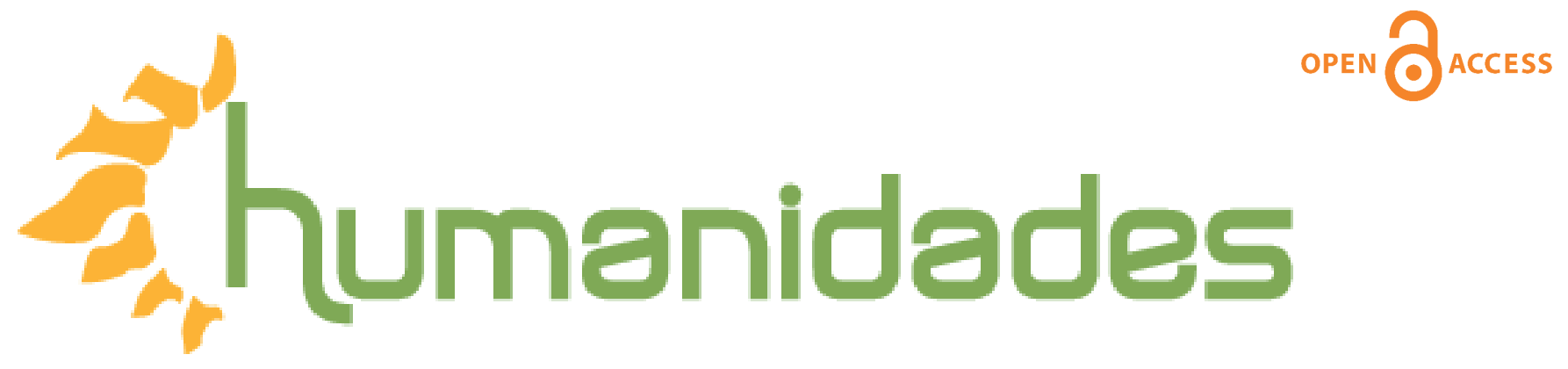

Revista de la Escuela de Estudios Generales, Universidad de Costa Rica

Julio-diciembre, 2018 •Volumen 8, número 2 • EISSN 2215-3934 •pp. 1-29

Recibido: 20-Abril-2018 Aceptado:30-Mayo-2018

\title{
Ogros en los malecus: sobre la elección y la res- ponsabilidad en la filosofía antigua malecu
}

DOI: http://dx.doi.org/10.15517/h.v8i2.33340

\section{Lic. Andrés Solano Fallas}

Universidad Estatal a Distancia, Escuela de Ciencias de la Educación, Centro de Investigaciones en Educación. Costa Rica.

Correo electrónico:sadsunsea@gmail.com

Todos los derechos reservados. Universidad de Costa Rica. Esta revista se encuentra licenciada con Creative Commons. Reconocimiento-NoComercial-SinObraDerivada 3.0 Costa Rica. Correo electrónico: humanidades@ucr.ac.cr / Sitio web: http://revistas.ucr.ac.cr/index.php/ humanidades 


\title{
Ogros en los malecus: sobre la elección y la respon- sabilidad en la filosofía antigua malecu
}

\section{Resumen}

El artículo tiene por finalidad explicar cómo son abordados los temas de la elección y la responsabilidad, a través de la figura del ogro (muérra), dada la trascendencia moral que implican en la narrativa malecu. En primer lugar, se desarrolla cómo la elección se manifiesta en las relaciones que los ogros mantuvieron con otros grupos de seres (los felinos, los seres humanos, el grupo familiar de los ogros, y los Dioses). En segundo lugar, se procede a tratar la relevancia de la responsabilidad desde dos puntos: desde el agente de las acciones, enfatizando que nadie puede presumir que su actuar no tiene consecuencias; y desde la responsabilidad de terceros, que plantea que los terceros no pueden suponerse ajenos a una situación, por el mero hecho de no ser afectados directos. Cabe señalar que este trabajo se realiza desde un abordaje de la filosofía analítica.

\section{Ogres in the malecu people: on the Choice and the Responsibility in theancient malecu philosophy}

Palabras claves: malecus, ogros (muérra maráma), elección, responsabilidad del agente, responsabilidad de terceros

\begin{abstract}
Responsibility are approached, by means of the figure of the ogre (muérra), given their moral transcendency implied in the malecu narrative. First, it develops how the Choice is manifested in the relationships that ogres held with other groups of beings (the felines, the human beings, the ogres' family group, and the Gods). Second, it proceeds to deal with the importance of responsibility from two points. The agent's actions, emphasizing that no-body can't presume his/her acting has no consequences. The third party responsibility, stating that third party can't suppose to be oblivious to a situation, just by not being directly affected by it.It should be noted that this paper is from the analytic philosophy approach.
\end{abstract}

Keywords: malecu people, ogres (muérra maráma), choice, agent's responsibility, third party responsibility 
Los malecu son un grupo indígena costarricense, perteneciente a la familia chibcha. Su nombre significa "nuestra gente" (ma-, nuestro/a; lecu, gente/ persona). Habitan el territorio Malecu; denominado por la oficialidad estatal costarricense como "Reserva Indígena de los Guatusos", a partir de su creación en 1976. Se ubica en el norte del país, en el cantón de Guatuso de la provincia de Alajuela. El territorio Malecu, de 2994 hectáreas, es un remanente de lo que alguna vez fue su territorio histórico, que posiblemente alcanzó las 1100 hectáreas (a mediados del siglo XIX), coincidiendo en gran parte con el área de la cuenca del Río Frío (ver Castillo, 2005a y 2005b). Actualmente existen tres palenques o comunidades: El Sol, Margarita y Tonjibe. Si bien son aproximadamente 1000 personas (Bolaños-Esquivel y González-Campos, 2010, p. 27), no todas viven dentro del territorio. Conservan su idioma, aunque no toda la población es bilingüe; además, existen variantes entre cada palenque. No obstante, se halla en un estado de decrecimiento, ya que "se encuentran adultos con una competencia únicamente receptiva en malecu o con una competencia activa reducida, niños y jóvenes con diferentes grados de competencia pasiva y productiva y ya no sobreviven sujetos monolingües en malecu"(Sánchez-Avendaño, 2011, p. 88).

Señalado lo anterior, el objetivo de este artículo es analizar dos temas que resultan de vital importancia en la filosofía antigua malecu11, a saber, la elección y la responsabilidad, según son presentados a través de las acciones de sus protagonistas, que tienen la notoriedad de ser "los malos" de la historia: los ogros (muérra maráma). Los ogros son parte del imaginario male$\mathrm{cu}$, que sirven no solamente para establecer códigos de convivencia a partir de sus historias, sino que también son medios para abordar temas como la libertad y muerte, aspectos que están implícitos en el acto deliberativo, como también para la promoción del razonamiento de las consecuencias del actuar, que conciernen al agente y en algunos casos a otros por las posibles afectaciones que se puedan ocasionar.

Para ello, se ha escogido como obra principal de trabajo, las Pláticas sobre ogros, que consiste en una serie de 18 textos que tratan "de incidentes ocurridos en época previa al cataclismo entre los ogros y los diversos grupos indígenas"(Constenla-Umaña, 2014, p. 9), los cuales son los pótos y los malecus2.

\section{Introducción}


El artículo se compone de dos partes, cada una remitiendo al tema en cuestión. En la elección se hará un recorrido a través de la interacción que sostienen los ogros con otros grupos de seres, con la finalidad de mostrar cómo en dicha interacción está presente el tema de la elección, por cuanto que se plantea que los ogros deciden cómo comportarse con cada grupo de seres. En la parte de la responsabilidad, de manera general, busca apuntar cómo en la filosofía malecu no se permite una elección exenta de aceptación de consecuencias del actuar, dado que los ogros pretendían des-responsabilizarse de sus actos.

Esta segunda parte tiene dos puntos: la responsabilidad del agente y la responsabilidad de terceros. En el primer punto, se enfatiza que el agente (el ogro), por más que niegue los efectos positivos o negativos, no solamente tiene que responder por lo que hace, sino tomar consciencia de ello, aunque sea por intervención heterónoma (en este caso, los Dioses). En el segundo punto, se hace hincapié en que la filosofía malecu no reduce la responsabilidad al agente, sino que la hace partícipe a terceros, si tienen conocimiento de la situación por la que esté atravesando el que requiera ayuda (en este caso, los pótos primordialmente, en segundo lugar, los malecus).

Vale indicar que en varias ocasiones se menciona al Dios jefe del panteón malecu, cuyo sobrenombre es "El de la Cabecera del (río) Nharíne". Los Dioses no eran referidos por su nombre propio -su enunciación estaba vedada, salvo ciertos casos-, sino por el lugar en el que habitaban, que usualmente era la cabecera de un río. En este trabajo se le abrevia simplemente como Nharine, en vez de hacer referencia a su sobrenombre completo.

Respecto a la citación -tanto para el texto principal como para otros editados por Constenla-Umaña- se ha optado por seguir la manera en que Constenla-Umaña editó los textos, en vez de utilizar APA. Cada texto está enumerado con números romanos, y las líneas con números arábigos, dado que esto permite una mayor facilidad de localización del pasaje. Por ejemplo, XI, 43-45, remite al texto número once, líneas 43 a la 45.

\section{Descripción de los ogros}




\section{Ogros en los malecus...}

Brevemente, los ogros son seres que habitaban el mundo físico antes de que los Dioses enviasen el cataclismo que borró a toda la primera humanidad. Se paseaban de noche por la selva, y de día dormían. No obstante, no eran animales ni seres humanos: son seres que físicamente se caracterizaban por ser demasiado altos, con enormes miembros sexuales (los ogros con penes grandes, las ogresas con vulvas grandes) que denotan unos deseos sexuales excesivos, con otros miembros corporales (tales como abultamientos en distintas partes, más de una flexión de codo en cada brazo, con varios pies a cada lado) que apuntan hacia un aspecto horrible.

\section{La Elección}

Solano-Fallas (2016) había comentado que la cosmología malecu presentaba un elemento ético importante, a saber, la posibilidad de poder elegir; consecuentemente, hacerse responsable de los efectos deseados o no deseados de la elección. En los textos sobre los ogros, vuelve a florecer este tema, con la particularidad de que en esta ocasión, no es a través de seres humanos que se despliega la relevancia que tiene la elección en los actos que se cometan, sino que los protagonistas son los infames agresivos, abusadores y devoradores de humanos: los ogros. Para estudiar el tema de la elección por medio de los ogros, se ha escogido como punto medular, la interacción o el contacto que los ogros tienen con los principales grupos de seres del mundo, según consta en la literatura malecu:
1) Los felinos (tafá maráma)
2) Los mismos seres humanos: malecus (malecu maráma), y principalmente pótos (póto maráma), así como los tarocafárrafas (tarocafárrafa maráma)
3) El grupo familiar de los ogros (muérra maráma)
4) Los Dioses (tocú maráma) que fueron los que crearon todo3

Se escogen estos grupos, debido a que los diversos vínculos de los ogros con ellos, denotan prácticas, así como actitudes diversas, que conducen s suponer que los ogros, si bien son violentos y brutales-desde la perspectiva 
humana-y que se conducen mal -desde la óptica divina-, han tomado en algún momento decisiones sobre cómo comportarse con cada grupo. Esto quiere decir, que a diferencia de un texto, por ejemplo, aristotélico en el que se explica cuál es el proceso deliberativo (cf. Aristóteles, 1111b 5 1113a 14), con los ogros no se investiga cómo llegan a decidir sobre cómo comportarse; sino que a través de sus conductas se muestra que hubo una elección, lo que a su vez resalta la importancia que los malecus le dedicaron a este tema.

Con los felinos parece no hubo mayor contacto que el habitar la misma selva, aunque con cierta desaprobación de lo que realizaban los ogros con los humanos. Los textos IV y VI son los únicos espacios textuales de este ciclo de pláticas en los que aparecen los felinos. En el texto IV se narra cómo los felinos no se interpusieron en el camino de un hombre se llevaba años perdido en la selva, y rescata un niño que había sido secuestrado por los ogros. Los felinos no le opusieron ninguna resistencia cuando el hombre decide matar a sus captores, y por orden de Nharíne, cría al niño. Tampoco atentaron contra su vida después de la muerte de los ogros, sino que incluso "se acompañaba amistosamente muy bien el hombre con los tigres" (IV, 41). Por su parte, el texto VI cuenta la historia de una mujer que fue secuestrada, así como también era violada constantemente por un ogro, del cual tuvo un hijo. Un día un felino llega, comunicándole a la mujer (cuando el ogro no estaba) que quiere ayudarla, por lo que dejará la piedra (que bloquea la cueva en la que es mantenida en cautiverio) "soplada" (VI, 39)4, pero que espere a que un hombre venga a terminar de remover la piedra. Estos dos textos muestran, en primer lugar, que los felinos no tienen intención alguna de atacar a los ogros. En el primer texto simplemente se apartan sin hacerse ningún daño, aunque la relación amistosa con el hombre, permite inferir que de algún modo estaban a su favor; mientras que en el segundo texto, un felino deja de ser espectador pasivo y decide ayudar, lo cual indica claramente que no estaba de acuerdo con el trato del ogro hacia la mujer.

En ambos casos, hay una desaprobación del comportamiento de los ogros -más evidente en el segundo texto-, pero se observa notablemente que no se traduce en atacar a los ogros, sino que se limita, en el primer texto, a no estorbar. En el segundo, en brindar una mínima ayuda aprovechando la 


\section{Ogros en los malecus...}

ausencia del ogro captor y violador, lo que a su vez parece denotar que el felino se estaba cuidando a sí mismo, porque no quería entablar un combate directo, dado que pudo haber aprovechado la situación para correr toda la piedra, pero no lo hizo, sino que solo le preparó el camino a otro para que terminara de rescatarla. Fuera de estos dos casos, no parece haber mayor relación entre ambos. Incluso en las Pláticas sobre felinos, no se menciona ningún contacto con los ogros, sino solamente la relación no-conflictiva que llegan a tener con los humanos antes del cataclismo por exhortación de Nharíne5. En lo que respecta a los ogros, tampoco se hace mención alguna de su trato con los felinos. Ni siquiera aparecen como sus depredadores, por cuanto que los ogros se alimentan de zopilotes (IX, 22-25), tepezcuinte (VI, 21) y otros animales inmundos, sin que el felino figurase en su lista alimenticia. De ahí que pueda afirmarse que su convivencia se limitaba, básicamente, a coexistir en un mismo espacio.

En cambio, con los humanos (malecus y pótos), la relación era completamente distinta. Los textos IV y VI dejan traslucir dos situaciones hostiles: el primero, en que los ogros secuestran niños, y que mataban hombres; el segundo, en que raptó y violó a una mujer. Estas dos situaciones no eran casos aislados, sino un comportamiento sistemático de los ogros. Los muérra maráma atormentaban a los humanos, principalmente a los pótos, y a veces a los malecus. A estos iban a robarles las esposas (IV, 4-7; XIII, 1-2 [en estas dos referencias son malecus]; X, 16; XIV, 27; XV, 59 [en estas otras son pótas]), lo que implica que eran abusadas sexualmente (I, 12-15; V, 5-15; VI, 16-17, 24-26; VIII, 32-33 [en todas estas referencias son malecus]), de las cuales algunas eran embarazadas (I, 24-25; V, 22; VI, 51-63 VII, 43; VIII 39; XII, 23; XIII, 44), mientras otras asesinadas y devoradas (III, 33-34; XI, 41; XV, 60; XVI, 55-56; XVII, 29; XVIII, 26, 107).

En otra serie de textos, "Pláticas de bótos"6, se vuelve a remarcar estos hechos, pero centrándose exclusivamente en los pótos. En términos generales, se indica que los ogros les hacían daño (I, 34; III, 38). De manera más concreta, que reiteradamente "los ogros les arrebatan y arrebataban las esposas" (II, 15), así como que se las comían (II, 16, 60-62; III, 19). Eran sumamente agresivos con los humanos, principalmente con los pótos, por cuanto que los ogros tendían a vivir en el mismo territorio póto7. 
En las Pláticas sobre ogros, se menciona en el texto XI la existencia de otros seres humanos antes del cataclismo, llamados tarocafárrafas, con la diferencia que eran igual de grandes que los ogros. El texto se concentra en explicar cómo los ogros $-\mathrm{o}$ por lo menos un grupo de ellos- fueron exterminados por los tarocafárrafas, por solicitud de Nharíne. De lo que puede extraerse del texto, los ogros y los tarocafárrafas no tenían contacto, no obstante, no parece que desconociesen mutuamente su existencia. Puede conjeturarse que la falta de interacción entre este tipo de ser humano y los ogros, se debió a que los ogros valoraron el hecho de que su estatura podía serles una amenaza, por lo que prefirieron no entrometerse con los tarocafárrafas. Esto podría respaldarse en el hecho de que Nharíne tomó nota de su gran tamaño, por lo que les pidió que los mataran, según se sugiere en el siguiente texto: "Se dice, pues, se dice, se dice que si no fuera por ellos, que eran tan altos, este... este... los tarocafárrafas,/ habría... ahora en efecto ogros" (XI, 37-38). Si Nharíne tomó ventaja de la estatura, no cabe dudar que los ogros también hubieran considerado a los tarocafárrafas un problema potencial, si se entrometían con ellos: su estatura no era algo que pudiese pasar desapercibido.

Por otra parte, en lo que corresponde a la relación familiar de los ogros, estos -desde la perspectiva de los Dioses- actuaban mal, ya que mantenían relaciones sexuales con sus parientes. De acuerdo al texto IX, 19-20, lo hacían con sus propias madres e hijas. Si bien la narración se limita a estos dos casos, no hay razón para suponer que las relaciones sexuales se restringían a estos dos grados familiares, ya que si se habían atrevido a fornicar con las ogresas que les dieron su vida, así como con las que fecundaron con otras ogresas, no podría explicarse por qué no lo harían con ogros/ esas de otros lazos sanguíneos como tíos, primos y abuelos, entre otros. Lo interesante es que estas relaciones sexuales incestuosas no las cometían porque hubiese escases de ogresas. De acuerdo a todas las pláticas, la raza "ogrezca" era bastante numerosa, sin que se aluda en ningún texto a que había menos ogresas. Incluso hubo uno, llamado Tablón de Laurel, que Nharíne desterró del territorio malecu, porque estaba reproduciéndose muy rápido (Texto X). Por lo que el motivo de sucumbir en incesto, no parece ser más que otro acto excesivo que cometían por iniciativa propia (cf. XIII, 10)

Finalmente, su interacción con los Dioses era irrespetuosa. Básicamente 
no obedecían ninguna de sus reglas, como la anterior de no fornicar con parientes, ni la de comer cosas inmundas. Además de Tablón de Laurel, hay otro ogro en el texto IX, llamado Tío Materno del Río, al cual Nharíne le reprocha su conducta, refiriéndose tanto a la falta de las reglas divinas como al trato con los humanos, en este caso, concretamente con los malecus -dado que era ahí donde vivía, antes de ser expulsado al territorio póto-. Con estos dos ogros, como con los demás, el problema era su mal comportamiento, así como su falta de obediencia.

Las cuatro interacciones que sostienen los ogros permiten observar, en primer lugar, que ellos no se comportan de tales modos con cada grupo, por algún tipo de predeterminación esencialista. En segundo lugar, que dicho comportamiento no es siempre malo, dado que esa es la impresión que parece darse, cuando se narra que los muérra maráma "no podían ser buenos ellos, los ogros" (I, 75). Los ogros no podían ser buenos, no porque estuvieran condenados o predeterminados, sino porque ellos habían decido no serlo. La manera en que se comportan, implica que sus actos se ejecutan con conocimiento y voluntariedad: dos condiciones que deben darse simultáneamente, debido a que son imprescindibles para calificar un acto como acto moral (Marlasca-López, 2004, p.66-67)

Préstese atención que con los felinos, su accionar es prácticamente nulo, ya que ni siquiera los consideran como objetos alimenticios. Debe tomarse en cuenta que los felinos, si bien son animales, son unas de las creaciones apreciadas por los Dioses, por lo que su carácter animal, no es sinónimo de inferioridad que induzca a que no se les deba dar su debida importancia en la cosmología malecu. Por el contrario, tenían mayor pre ponderancia antes del cataclismo -y de alguna manera, la siguieron teniendo como aliciente moral después del cataclismo (Solano-Fallas y Herrera-Valenciano, 2017). Por tanto, los ogros al no entrometerse con ellos, es un indicador de que hubo una decisión de no tratar con los felinos. Esta falta de relación en ningún momento se expresa que se deba por un mandato de Nharíneo algún otro Dios, lo que permite reforzar la idea de que los ogros dispusieron no lidiar con ellos. Cuál haya sido el motivo, se desconoce. Lo que interesa es que su co-existencia sin lazos, implica que los ogros eligieron no atormentarlos; contrario lo que sucede con los humanos pótos y malecus. 
Con los humanos tarocafárrafas, básicamente se presenta la misma situación que los felinos: no existe interacción, no porque hubiese algún mandato, sino por la prudencia que los tarocafárrafas podían hacerles frente; que efectivamente les hicieron, cuando un grupo de ogros fue asesinado por estos seres humanos. En cambio, la elección es muchísimo más evidente con los humanos malecus y pótos. Particularmente con los pótos, estos son humanos de muy baja estatura, por lo que resulta difícil que fuesen concebidos como un peligro para los ogros; máxime si se tiene en consideración que "los votos no se desquitaban en nada" (XIV, 32). Además, según la apreciación que tienen los malecus de ellos, "no...no... son listillos los votos" (XIII, 31), por lo que no constituyen un riesgo, como si lo suponían sus congéneres tarocafárrafas.

Puede afirmarse que el actuar "ogrezco" es típico de una conducta agresiva, debido a que el daño -o distintos tipos de daños: psicológicos, emotivos, físicos, entre otros- que un sujeto (o sujetos) recibe por parte de otros (u otros), no es un hecho aislado, sino que existe una "tendencia a comportarse agresivamente en las distintas situaciones (Berkowitz, 1996) a atacar, faltar el respecto, ofender o provocar a los demás intencionalmente" (Carrasco-Ortiz y González-Calderón (2006, p.9)8. En el caso de los ogros, estos muestran una agresividad con los pótos, ya que en sentido estricto no son una amenaza a su existencia: "los pobres votos no se desquitaban en nada" (XIV, 32). Los textos del XVI al XVIII, enfatizan en que los pótos eran víctimas que vivían atormentadas por los ogros, sin que les hubiesen hecho algo para generar su crudeza. Tampoco en los textos se menciona que ambos seres compitan por recursos, ya que la selva parece proveer lo suficiente para las dos especies. Sin embargo, los actos agresivos, asesinos, de violación, y devoración, entre otros más, se debe a que así lo han querido hacer. En lo concerniente a la violación, habían ogresas suficientes para reproducirse, en caso de que ese fuese el objetivo; por lo que recurrir al abuso sexual de mujeres no se explica más que por una elección para gozar, salvó algunos casos en que explícitamente se quiere incrementar el número de ogros, lo que a su vez implica que igualmente hubo una decisión cuando se raptó a una mujer. Esta libre voluntad muestra que actúan de mala manera, no porque estén predeterminados. Su accionar con los humanos es otro indicador de que hay elección: con unos (tarocafárrafas) no deciden ni acercárseles, con otros (pótos en primer lugar, malecus en segundo) hay todo un despliegue 


\section{Ogros en los malecus...}

de agresividad que escala a violencia9. De manera similar puede decirse que con el incesto hubo un acto deliberativo. Según se explicó, los textos no permiten concebir la posibilidad de que hubiese escasez de ogresas. Entonces, si habían las ogresas suficientes para gozar sexualmente o reproducirse, no tiene sentido que acudieran a sus familiares. Si estuviesen predeterminados a esas prácticas sexuales, es decir, que no tuviesen la posibilidad de elegir actuar o no incestuosamente, tampoco tendría sentido que Nharíne les insista en que no se continúen comportando de tal manera, ni mucho menos que les reclame: "Perversamente... fornicáis... con... con... vuestras madres,/ perversamente fornicáis con vuestras hijas” (IX, 19-20). La única explicación razonable y posible, consiste en que había sido una elección.

Lo mismo cabe decir de la falta de obediencia hacia los Dioses. Cuando Nharinese comunica con los ogros, lo hace de manera exhortativa. Por ejemplo, al ogro Tío Materno del Río, le expresa, en relación a su comportamiento, que "no quiero que os conduzcáis mal" (IX, 9). No hay duda que establece reglas de conducta en orden a que exista equilibrio en el mundo, empero, sus reglas no son constituyentes naturales de los muérra maráma. Como se indicó con los felinos, Nharíne les pide que dejen de asolar la selva, a los humanos les solicita múltiples veces que actúen bien, así como a los ogros. Si estas reglas estuviesen inscritas en la manera de ser de los ogros, sería complicado -si es que no difícil e imposible- de explicar por qué se comportan de manera distinta a como fueron creados. Por ello, su mal comportamiento no corresponde más que a una elección a no seguir las reglas de los Dioses, según se hace manifiesto en la queja al mismo Tío Materno del Río, cuando Nharíne le comenta: "porque te conduces mal, os he estado observando" (IX, 27). Nótese como el elemento de observancia, en el caso de este ogro, es un indicador de su mal comportamiento ha sido constante, y por decisión propia.

Por consiguiente, los malos actos "ogrezcos" con los humanos malecus y pótos, como también entre sí mismos con sus parientes, su desobediencia a los Dioses, así como su carencia de contacto con los felinos y con los humanos tarocafárrafas, conducen a plantear que en la tradición malecu existe una importancia al tema de la elección. Con los seres de cada grupo, tuvieron la capacidad de decidir cómo interactuar convivencialment, por lo que no 
existe ningún tipo de determinismo preestablecido que niegue la voluntad.

La elección es un punto decisivo que la filosofía malecu rescata y proyecta en sus narraciones, debido a que está consciente que un ser humano no puede vivir, sin percatarse que cada acción tiene uno o más efectos. Tomar una decisión implica marcar tanto la vida propia, como la vida comunal, ya que en el caso de los malecus, una elección personal puede tener consecuencias en otros. De ahí que los malecus concibieran la relevancia de hacer a los seres humanos responsables de los efectos de sus actos, puesto qué sentido habría en establecer reglas para asegurar una convivencia equilibrada en el mundo, si los propios sujetos de actuación ya se encontraban predeterminados desde su creación.

\section{La responsabilidad}

Vinculado a la elección, se encuentra otro tema de importancia capital en la filosofía malecu, a saber, la responsabilidad, entendiendo por esta como "sencillamente la capacidad que tiene un sujeto de responder por sus propios actos, sean de la especie que fueren, incluso en la hipótesis de que las consecuencias de sus actos sean dolorosas y no fueran exactamente las queridas y previstas" (Marlasca-Ló pez, 2004, p.83). Para la tradición malecu, no puede existir ningún ser humano -como tampoco ninguno de los principales grupos de seres que se indicaron con anterioridad- que pueda des-responsabilizarse convenientemente de lo que hace, o permanecer indiferente ante lo que le suceda a otro. Los textos sobre los ogros entrañan un enriquecimiento ético, en el tanto de que no solamente se explayan sobre el deber que tiene cada persona de asumir las consecuencias negativas y positivas de su actuar, sino que también plantea que la responsabilidad no se reduce al agente (en este caso a los ogros), sino que en diverso grado atañe a terceros.

\subsection{Responsabilidad del agente}

Con los ogros puede apreciarse como su comportamiento tiende a desentenderse de sus acciones, creyendo que en modo alguno estas tendrían alguna repercusión. Esto se nota en las relaciones que mantenía con los distintos 


\section{Ogros en los malecus...}

grupos: en principio con los felinos y los humanos tarocafárrafas no tenían conflictos; con los humanos pótos y malecus, a pesar de que estos se vengarán a veces, no representaban realmente un peligro, menos los pótos que eran más pequeños que los malecus; sus parientes ogros parecían no oponer objeción a la incestuosidad; y las exhortaciones, así como castigos, de los Dioses eran fútiles. Sin embargo, los textos son enfáticos que la elección no exime de la debida responsabilidad de los actos.

Cabe preguntarse -aunque sea especulativamente- por qué los ogros creyeron que podían actuar con impunidad, sin asumir las consecuencias de sus acciones, particularmente contra los pótos. No es posible saberlo a falta de evidencia textual, aunque puede conjeturarse que los ogros no enfrentaban la angustia de la muerte.

Brevemente, la muerte es un tema de vital importancia en la tradición malecu, porque para los seres humanos puede generar zozobra según el tipo de muerte que se tenga, dado que existe una vida post-mortem. Hay dos tipos de muerte: la buena muerte y la mala muerte. La primera consiste en morir por causa natural o alguna enfermedad, lo cual es un indicador de que en vida fue una buena persona, lo que quiere decir, que cumplió con las reglas dietéticas, sociales y sexuales de los Dioses. La buena muerte implica que se hizo meritorio a que su alma pase a morar en las casas de los Dioses, que se encuentran en las cabeceras de los ríos. Por su parte la mala muerte tiene dos subtipos: el que muere mordido por una serpiente o el que muere de una manera trágica. Ambas son indicadores de que la persona, en vida, no se guiaba conforme a las reglas divinas, por lo que a su alma le corresponderá ir a morar con los Diablos (maíca maráma) y sus sequitos. Quien tuvo el primer subtipo de mala muerte, irá al cielo a ser torturado por el Diablo Oronhcafá; en cambio, quien murió de manera trágica, irá con Lhára (o Jára), en el río Tilhacalí (lugar terrenal de tormentos).

A pesar de que los textos no indiquen que los ogros no tienen almas, de acuerdo a Constenla-Umaña (1993, p.30-31), el alma es propio de los seres humanos. De esta manera, el ogro al no tener alma, no debió haber tenido la preocupación de tenerse que comportar, de manera no-conflictiva con los humanos pótos y malecus, así como no-incestuosamente con sus parientes, para acceder a una buena muerte. El ogro se creía libre de responsabilidad, 
ya que creyó que jamás pagaría por sus acciones en la vida post-mortem, por cuanto que al morir, simplemente cesaría de existir. Incluso cuando algunos de ellos son asesinados por iniciativa humana o divina, no se indica que haya alguna retribución posterior a la vida.

Los ogros son el ejemplo paradigmático que, tanto la buena muerte como la mala muerte, pierden eficacia ante seres que no les provoca la más mínima intranquilidad, lo que implica que la muerte no es el único mecanismo por el cual pueda estimularse a que se tome consciencia los sus actos propios, por ende, que elija y actúe responsablemente1 $\square$. En el caso de los ogros, los Dioses llegan a la conclusión -después de varios intentos fallidos- de que darles muerte no ayudaba en nada. En distintas ocasiones, los masacran personalmente (texto I), o bien enví an humanos a hacerlo (en el texto XI son los tarocafárrafas, mientras que el XII, son malecus acompañados de pótos), pero los ogros continuaban comportándose irresponsablemente. Por lo que Nharíne decide que la única manera de conseguir que, efectivamente, tomen conciencia de su mal comportamiento, es el exilio.

El jefe de Dioses ordena que los ogros sean expulsados de los territorios malecus y pótos, y enviados al "extremo de la gran extensión de agua, al otro lado del agua" (XVIII, 69). Esta deportación logró, finalmente, causar un impacto en los ogros, por cuanto que, según los textos XIV al XVIII, tanto los ogros maduros como las ogresas maduras, los ogros hijos y las ogresas hijas, y los ogros abuelos, en el momento de su captura se inquietaron demasiado respecto a cuál sería su suerte: durante el periodo de captura y contención, se angustiaron en si los iban a matar, y cómo los matarían, dado que Nharíne encargó a los pótos que los vigilasen, por lo que sospechaban de una venganza. Es decir, en este último momento, tuvieron miedo a morir, pero no a la muerte en sí misma.

Entiéndase que no les preocupaba la vida del más allá, ya que no tenían alma, sino en que al estar todos capturados, se preocupaban si los humanos se iban a ensañar con ellos. Pero Nharíne, en algunos casos personalmente, o por medio de su hija Jafára, les aseguró que nadie los mataría. Probablemente tomó esta decisión, porque se percató de que la muerte no sirve con ellos, ya que no se les podría reprochar ni ajusticiar por sus actos en 
una vida post-mortem que no pueden tener. El exilio fue prácticamente un tormento para los ogros, dado que representaba la imposibilidad de poder volver a la selva, es decir, su hogar:

He aquí vine a dejaros aquí,/ aquí os... os asentaréis en cuevas de piedra, en cuevas... de cascajo./ Aquí viviréis en cuevas de piedra, aquí viviréis en cuevas de tierra,/ imposible que regreséis, que se os vuelva a ver./ Sucede que para siempre... aquí vinisteis a perderos, a quedaros donde estáis (XIII, 21-25).

Mientras los iban a dejar en balsas, "se dice que de manera extraña daban alaridos" (XVIII, 84), y que "ya lloraron y lloraron" (XVIII, 103). A pesar de que se quejaban a gritos (XVIII, 96, 101-102), parecen haber comprendido el porqué de su situación. De esta manera, se les imputó sus acciones, ya que el exilio representaba un castigo que tenían que pagar, por sus acciones violentas contra los pótos11, lo que muestra que ningún acto queda impune. Ellos eligieron actuar de cierta manera con unos y distinta con otros, creyendo que la elección no tenía consecuencias, por ende, que podían des-responsabilizarse. No obstante, el exilio es sintomático que ninguna acción carece de reacción ética: todos los seres, sin importar quien sea, deben responder ante sus actos. En el caso de los ogros, estos decidieron no hacerlo por sí mismos, por lo que de manera heterónoma, se les tuvo que mostrar que no podían permanecer impunes.

\subsection{Responsabilidad de terceros}

Como se había indicado previamente, en las narraciones malecus surge una cuestión éticamente interesante. Si bien el tema de la responsabilidad concierne en primera instancia, y con mayor incidencia, al agente por cuanto que ha sido él/ella quien decidió qué hacer, las Pláticas sobre ogros plantean que los terceros también tienen su cuota de responsabilidad ante la situación del otro; máxime si tienen algún conocimiento de lo que está sucediendo. Partiendo del planteamiento de Rodríguez (2010), esta responsabilidad es "asimétrica", en el sentido de que el tercero que llega a percatarse de la situación del otro, tiene un deber moral de asistirlo, pero teniendo en cuenta que solo podrá ayudar, según sus posibilidades, debido a que es imposible 
"poder satisfacer todas las demandas morales que surgen de los múltiples otros que encontramos en nuestra vida" (Rodríguez, 2010, p.122). Por ello, no puede demandarse que cada tercero tenga el mismo grado de responsabilidad, ya que dependerá tanto del conocimiento que posea de la situación, como también de lo que pueda realizar.

En el caso de los felinos, parecería que no había ninguna responsabilidad que les atañese, porque en principio, no mantenían ninguna vinculación con los ogros. Sin embargo, en los textos IV y VI, se hace patente la desaprobación de algunos elinos respecto de la manera en que trataban a las mujeres. Esto implica que, si bien no había una interacción entre ambas especies, sus actos no pasaban desapercibidos. Los dos textos en cuestión -como el resto de las narraciones en Pláticas sobre ogros y Pláticas sobre felinosno muestran un reproche o queja directa de los felinos a los ogros, empero permiten inferir que una acción no puede pretenderse impune. Este punto resulta éticamente compelente, debido a que plantea que, aún si una persona no es la afectada directa, no puede permanecer impávida ante la suerte de un congénere. Aunque decida no enfrentar directamente al agresor, por lo menos ha de realizar una acción, por más mínima que sea, en beneficio del que esté necesitado de ayuda.

En los textos puede verse cómo el felino no tiene la intención de hacer al ogro responsable de sus hechos, pero las acciones ogrezcas compelen a los felinos a tomar algún partido. En el texto IV el grupo de felinos deciden, por lo menos, no interferir en el rescate del niño, lo cual puede resultar éticamente cuestionable por no haber hecho mayor cosa que no estorbar. En todo caso, se oponen a los ogros: bien pudieron haber matado al hombre antes o después del rescate, e incluso al niño. Con el texto VI, la oposición es más evidente que la anterior, al "soplar" la piedra y darle instrucciones a la mujer secuestrada.

Por tanto, estos textos muestran dos asuntos. El primero, que las acciones de los ogros generan algún tipo de reacción, es decir, que ninguna acción (sin importar quien la ejerza) puede suponerse que no impactará de alguna manera en la conciencia de otro ser o grupos de seres. En relación al anterior, el segundo asunto indica que la responsabilidad no es una cuestión 


\section{Ogros en los malecus...}

que solo incumba al agente (en este caso al ogro), sino que la persona o colectivo impactado (en este caso, los felinos de los textos IV y VI), una vez que ha tenido conocimiento de alguna situación, tampoco puede desligarse y pretender que por no tener contacto con el agente, no tiene algún tipo de obligación hacía con su congénere que requiere ayuda.

De manera similar ocurre con los humanos tarocafárrafas. Una primera impresión haría conjeturar que no tienen por qué inmiscuirse en un asunto de agresión física y sexual, de sus congéneres humanos, por el hecho de que no son ellos los afectados. Sin embargo, no era posible que las acciones de los ogros fuesen ignoradas, ya que de algún modo, sería como desconocer la existencia misma de los ogros, quienes - a juzgar por las narraciones- eran notorios; máxime que Nharíne les solicita a los tarocafárrafas que los maten. Este asesinato, perpetrado contra los ogros, es sintomático de que los tarocafárrafas, a diferencia de los felinos, decidieron no solamente tomar partido, sino ser plenamente activos. Podría decirse que asumieron la tarea divina con responsabilidad, dado el grado de eficacia y eficiencia con que la cumplieron, debido a que no podían permitir que la atrocidad contra los otros humanos (principalmente los pótos) continuase.

\section{Conclusión}

Las Pláticas sobre ogros resultan de un interés ético vigente. La elección realmente moral, es decir, no coaccionada, presupone que el agente tiene conocimiento relativamente pleno de lo que realiza, y se halla libre para hacerlo. En otras palabras, conoce el objeto de su acción, las circunstancias en que lo efectúa, y la intencionalidad. Como pudo observarse, las interacciones de los ogros con los demás grupos de seres, si bien no explicaban cada uno de estos elementos, los daban por supuestos, debido a que el ogro no actuaba guiado por un determinismo. La indiferencia que sostuvieron con los felinos y los tarocafárrafas, así como la crudeza con los otros humanos, junto con la incestuosidad dentro su propio grupo familiar, eran actos morales que derivaban de una decisión.

En lo concerniente a la responsabilidad, esta implica que se asuman las consecuencias, directas o indirecta, de lo que sea realice, a su vez,presuponiendo 
conocimiento y voluntariedad, ya que no se podría adjudicar o imputar a alguien la autoría de un acto si lo ejecutó bajo ignorancia y/o coaccionado (Marlasca-López, 2004, p.66). De lo contrario, se trataría de un acto instintivo y carente de moralidad. Los textos, mediante la figura del ogro, dejan en claro que si alguien actúa libremente y con comprensión de lo que hace, no puede evadir las consecuencias de lo que ha elegido cometer. No obstante, la filosofía malecu antigua no se limitó a centrar la responsabilidad en el agente, sino que la hace extensiva a aquellos terceros que tengan algún conocimiento de lo que esté aconteciendo, y que, estando en la medida de sus posibilidades, puedan hacer algo al respecto. De esta manera, la capacidad de responder por las consecuencias del accionar se convierte en esta filosofía, en un asunto de dos partes (two-party): el agente y los terceros.

El involucramiento de terceros, y su respectiva atribución de responsabilidad, es un resultado lógico de esta filosofía, por estar en correspondencia con su concepción de mundo como gran casa (ú cájayá). En las pláticas cosmogónicas, Laca Majifijica (La Transformación de la Tierra), se considera que el mundo es efectivamente una casa, lo que conduce a pensar que no se trata de "cosa cualquiera", sino del soporte o ámbito en el cual se desarrolla la vida humana en sus distintas dimensiones. Por lo tanto, habitar una gran casa denota la coexistencia de varios seres en un mismo lugar, por lo que no pueden pretender ignorarse mutuamente; además, implica que los habitantes deben seguir algunas pautas conductuales que garanticen la sana convivencia grupal, debido a que en la filosofía malecu, si bien cada ser humano puede distinguirse de otro, no es un ser "tipo átomo" (aislado), sino uno que vive en una misma casa, con otros miembros, por lo que el actuar de uno puede afectar el de otros.

Por ello, es relevante recalcar, así como recordar, que la responsabilidad está vinculada con la elección. En el caso del agente, resulta clara y manifiesta por ser él/ella quien está llevando algo a cabo; en el caso de los ogros, dañar a los pótos y a los malecus, como también desobedecer a los Dioses. En cuanto a los terceros, al tener conocimiento y voluntad, no puede excusarse de que no le compete o incumbe por no ser afectado directo, por tanto, no puede alegarse que no era su elección, por no tratarse de un asunto que le concerniera. El hecho de que alguien quiera des-responsabilizarse,implica 


\section{Ogros en los malecus...}

que realiza una elección, por cuanto que conoce la situación de lo que está acaeciendo, y su libertad para actuar no se está viendo totalmente restringida. Por ello, es que el actuar de los felinos (en estas pláticas), a pesar de la "asimetricidad" que condiciona su intervención, resulta éticamente cuestionable, dada su escasa participación, mientras que la de los tarocafárrafas resulta loable y edificante de la responsabilidad de terceros, ya que al parecer ninguno de los dos tipos de seres, estaba ni total o parcialmente impedidos para actuar.

\section{Notas}

1. Los términos de "malecu antiguo" y "filosofía malecu antigua", tienen por intención establecer una diferenciación con respecto al malecu "actual", dado que este lamentablemente se ha visto víctima de una fuerte aculturación, que inició principalmente con la guerra de los huleros nicaragüenses en la segunda mitad del siglo XIX, continuando con la intervención del Estado costarricense y la religión cristiana a partir de la segunda mitad del siglo XX. Sin pretender ahondar en detalles, el malecu actual, si bien busca mantener los elementos culturales que lograron sobrevivir, ha sido sujeto del desplazamiento de aspectos importantes de su cultura, como los modos de producción, la paulatina extinción en la que se encamina su idioma, la pérdida de sus creencias religiosas reemplazadas o sincretizadas con el cristianismo, entre otros factores.

El contenido de la transmisión oral de sus pláticas tiende a remitir a momentos, por lo menos, bastantes previos a los dos fuertes contactos anteriores que tuvieron con la cultura no-indígena. Cabe citar a Sánchez-Avendaño (2015) quien sumariza el contacto con los españoles conquistadores y colonizadores, es decir, antes del siglo primer contacto fuerte en la segunda mitad del siglo XIX:

En suma, tal parece que los malecus lograron mantener su cultura vernácula durante toda la Colonia y los primeros años de la República debido a su aislamiento con respecto a los grandes asentamientos españoles en el Valle Central y la Península de Nicoya. 
Es probable que su contacto con los colonizadores y mestizos fuera escaso o nulo al menos hasta la segunda mitad del siglo XIX, a juzgar por la falta de referencias sobre su existencia en los documentos de las distintas épocas y por lo datos presentes en la misma tradición oral de este pueblo (Sánchez-Avendaño, 2015, p.88).

El que se haya preservado la cultura vernácula antes de la primera mitad del siglo XIX, implica que la filosofía que deriva de esta, también tuvo que haberse conservado hasta que el sistema de producción, el orden social y el marco axiológico entrasen en choque. Por consiguiente, tanto el malecu antiguo, como la filosofía antigua malecu previos a la primera mitad del siglo XIX, son distintos del malecu actual como también de una posible filosofía que pueda pensarse a partir desde el colectivo grupal en su nueva situación social-económica-religiosa. Finalmente, en aras de la aclaración, debe puntualizarse que por "antigüedad malecu" no se pretende, ni se presupone, una visión idealizada de dicho pasado. Esto viene a colación, porque el malecu actual añora su pasado, debido a la nostalgia que le genera la pérdida de elementos de su cultura, lo que ha conducido a que recuerden la época previa al primer contacto con los no-indígenas, como un tiempo en el "que el pueblo malecu conservaba su presunto estado original y en los que la homogeneidad grupal era supuestamente absoluta"(Sánchez-Avendaño, 2015, p.187).

2. Estos textos originalmente pertenecen a la tradición oral malecu, pero gracias a la labor de Eustaquio Castro (informante malecu) y Adolfo Constenla-Umaña, han sido editados. Siguiendo la catalogación de Constenla-Umaña (2011), en la tradición oral existen varios géneros. El que concierne a este artículo es el género de pláticas tradicionales, en el que se abordan distintos elementos que dan cuenta del pasado histórico-cultural del pueblo, pero sin que se realice de manera cantada. Dado que estos elementos fueron agrupados por los malecus en series de textos, según un hilo temático o teniendo a unos seres en concreto como protagonistas, se crearon ciclos de pláticas que versan sobre cada uno. Por ejemplo, están los ciclos de las pláticas sobre los ogros, sobre los felinos, sobre los duendes, sobre los indígenas pótos, sobre la creación de la tierra, entre otros. A estos ciclos, Constenla-Umaña 


\section{Ogros en los malecus...}

los consideró como subgéneros de las pláticas tradicionales, ya que cada uno aborda elementos del pasado, de manera más o menos separada uno del otro (pero manteniendo una relación interdependiente).

En cuanto a su finalidad, era principalmente didáctica, dado que trasmiten creencias, valores, historia del pueblo malecu, entre otras informaciones (Constenla-Umaña, 1992), debido a que en algunas ocasiones se hace explícito que se cuenta estas historias a los niños para que sepan comportarse. En lo que concierne al acto de ser relatado, Constenla-Umaña indica que "nunca se cuenta más de tres veces a la misma persona, pues se considera que, si hay auténtico interés, ese número de repeticiones deben bastar para la memorización” (2011, p.9).

Con base en lo anterior, las Pláticas sobre ogros, y otras que se utilizaron en el presente trabajo, corresponden al género de pláticas tradicionales. En el caso de Pláticas sobre ogros, por consiguiente, al subgénero de pláticas tradicionales sobre ogros, tal como se desprende del título con el cual fueron editados. De manera análoga, cualquier otra serie de pláticas a la que se haga mención, el título denotará su subgénero.

3. En relación a los nombres en malecu, cabe indicar que el término marámaque aparece en cada uno, es un plurificador, por lo que tafá, malecu, póto, tarocafárrafa, muérra y tocú, por sí solos, son sustantivos singulares, que quieren decir felino (o tigre, dependiendo de algunos casos), malecu (a veces se le traduce como indígena), póto, tarocafárrafa, ogro y Dios, respectivamente. Sobre su empleo en este trabajo, para lo que atañe a los felinos, ogros y Dioses, se optó por utilizarlos en español; con la excepción de "ogros", que a veces se alterna con su original en malecu.

En el caso de los nombres de los grupos humanos, se prefirió utilizarlos tal cual en manera singular, y castellanizar su plural añadiendo una "s". En el caso del malecu, porque sería confuso traducir "nuestra gente"; además, valerse del término "indígena" para traducirlo, considero que diluye su impronta como grupo indocostarricense concreto. En cuanto a los términos pótos y tarocafárrafas, se mantienen en su original y respectiva castellanización plural, debido a que se desconoce sus significados. 
Explicado lo anterior, vale hacer algunas referencias puntuales para comprender quienes son los felinos, los humanos, los ogros y los Dioses en el imaginario cosmovisional malecu. Los felinos son animales que gozan del aprecio de los Dioses, y fueron creados principalmente por Nharíne. En razón de dicho aprecio, no son animales inferiores, como los demás que se mencionan en los textos, sino que están por encima de la "escala animalia alimenticia”, máxime que antiguamente se creía que podían transformarse en humanos. Estos seres son igualmente importantes en la filosofía malecu, debido a que abarcan temas como la muerte, e igualmente, la responsabilidad y la elección. Para un abordaje filosófico, puede verse Solano-Fallas y Herrera-Valenciano, 2017.

Sobre los humanos, la cosmogonía malecu plantea que todos fueron creados por Nharíne, en la primera ocasión que se formó el mundo. Es importante señalar que los dos primeros son históricos, mientras que el último parece ser mitológico. En cuanto a los pótos propiamente, eran indígenas ramas, pertenecientes a la familia chibcha, por lo que estaban emparentados con los malecus, aunque sin ser del mismo grupo. De acuerdo a Ibarra-Rojas (en Constenla-Umaña e Ibarra-Rojas, 2014, p.114), en el siglo XVI ellos vivían en las tierras más cercanas al Lago de Nicaragua y al río San Juan, pasando por el río Frío y llegando a pasar el río Sarapiquí; por lo que solo habitaron parte de la cuenca del Río Frío. Según Constenla-Umaña (2014, p.135; nota \#2), los malecus parecen acordarse de ellos como vecinos específicamente de la zona circundante al curso inferior del río Frío, como consta en las pláticas.

Por su parte, a los tarocafárrafas, se les puede considerar actualmente como seres mitológicos, no obstante para el imaginario malecu se trataron de un linaje patrilineal que alguna vez existió (Sánchez-Avendaño, 2015, p.58, nota \#15). La narrativa malecu da cuenta de que Nharíne les concedió una buena muerte previa al cataclismo, porque debido a su buen comportamiento, el Dios jefe no quería que sufrieran las consecuencias devastadoras de la transformación de la tierra. Estos humanos eran descritos como seres de gran altura, prácticamente gigantes. De los ogros ya se realizó una descripción breve. Lo que cabe indicar es que los textos no explican su origen. Se desconoce de dónde provienen, o si fueron creados por los mismos Dioses. 
En cuanto a los Dioses, para mayor detalle puede remitirse a la reseña en Solano-Fallas 2016. Rápidamente, provienen de un lugar desconocido. El primero en llegar fue Nharíne, seguido de su futura expareja, la que habita en el Aóre, y demás Dioses. Nharíne crea o forma la flora y fauna, como también a los humanos, no obstante, Aóre no estaba contenta con lo que ella creaba, dado que solo producía animales inmundos; razón por la cual insiste a Nharíne que transformen la tierra, es decir, que la destruyan. Luego de múltiples insistencia, accede cuando ve a los malecus realizar orgías heterosexuales y homosexuales, además incestuosas. El trato con Aóre había sido que ella crearía todo en la segunda ocasión, pero fracasó, debido a que solo logró producir (nuevamente) cosas inmundas y marchitas; por lo Nharíne vuelve a crear todo. Distintos textos, en especial los del ciclo cosmológico, tratan de asignar el acto creativo a Nharíne, aunque hay algunos textos en que se muestra que otros Dioses hicieron su parte.

\section{Podría entenderse esta expresión como "floja".}

5. En líneas generales, según las narraciones sobre Pláticas sobre felinos, los felinos asolaban las selvas (II, 38; VII, 7-10; VIII, 57; IX, 75-76), pero Nharíne les dice que no pueden continuar comportándose de esa manera, dado el daño que estaban causando entre los humanos (I, 6, 26,39, 45, 80), y que corrían el riesgo de ser atacados, si los humanos actuaban en defensa propia (I, 46-47, 81) o en venganza (VIII, 61-62); por lo que llegaron a compartir el espacio vital de existencia sin conflictos. Para mayor detalle y otros temas que contienen estas pláticas, ver Solano-Fallas y Herrera-Valenciano (2017).

6. Estas pláticas constan de ocho textos que proyectan la visión estereotipada que tenían los malecus respecto de los pótos. A diferencia de los ciclos narrativos en Pláticas sobre ogros, en que se presupone que el malecu ya sabía de antemano quien era el póto, en estas otras narraciones se brinda explícitamente la percepción que tenían de ellos. Asimismo, si bien mencionan brevemente episodios con los ogros, algunas pláticas se centran en el contacto que mantenían con los malecus.

Por otra parte, como puede notarse, el título de las pláticas denota una diferenciación en la grafía (y pronunciación) que utilizo en este artículo. 
El término "póto" corresponde a como lo pronuncian los mismos malecus en su idioma, mientras que su grafía con " $b$ "-y en algunos casos con "v"se debe a como lo escribían (y proferían) los españoles en sus crónicas de la época de la conquista (Ibarra-Rojas en Constenla-Umaña e Ibarra-Rojas, 2014, p.114; y Constenla-Umaña, 2014, p.4). Sobre su grafía en este artículo, utilizo la letra " $\mathrm{p}$ " cuando mi persona, en calidad de autor, se refiere a dicho grupo indígena; no obstante, si estoy reproduciendo alguna cita directa, respeto la grafía de la fuente, sea que esta venga con la letra "b" o la "v".

7. Originalmente, los ogros habitan el territorio malecu, pero a causa de sus acciones, Nharíne los expulsó al territorio póto (textos IX y X). Se desconoce porqué los traslado de un lugar a otro, ya que realmente no fue una solución, sino una trasferencia de problemas: en vez de que los malecus tuvieran que lidiar con los ogros, se los pasó a los pótos. En todo caso, Nharíne y los Dioses se dan cuenta de este inconveniente, por lo que deciden buscar otra solución.

8. Para Carrasco-Ortiz y González-Calderón (2006, p.9), la diferencia conceptual entre "agresión" y "agresividad", radica en que el primer término remite a "un acto o forma de conducta "puntual", reactiva y efectiva"; mientras que el segundo término a la tendencia antes menciona en la cita del cuerpo del texto.

9. Tanto en un plano conceptual, como cotidiano, los términos "agresión" y "violencia", tienden a confundirse, debido a que ambos remiten a conductas yhechos que causan daños en uno o varios sujetos. Siguiendo a Carrasco-Ortiz y González-Calderón (2006, p.10), “[e]1 término violencia se suele emplear para referirse a conductas agresivas que se encuentran más allá de lo "natural", en sentido adaptativo, caracterizadas por su ímpetu, intensidad, destrucción, perversión o malignidad, mucho mayores que las observadas en un acto meramente agresivo". En el caso de los ogros, no hay duda de que cometían agresiones, pero también hechos violentos, por ejemplo, cuando mataban mujeres por gusto.

10. En lo que respecta a los seres humanos, la muerte en sí misma parece 


\section{Ogros en los malecus...}

ser el mecanismo por excelencia, aunque también se vale de felinos paraanunciar a una persona su mal comportamiento, para que tenga la posibilidad de cambiar (Solano-Fallas y Herrera-Valenciano, 2017). Respecto de los ogros, no puede decirse que los felinos tengan la misma función con ellos, por cuanto que no mantenían ningún vínculo.

11. Los textos parecen enfatizar que el exilio se debió más por lo que le hacían a los humanos, que a lo que hacían entre sí mismos, por lo que sus hábitos alimenticios (que eran inmundos) y sus relaciones incestuosas, parecen no haber sido considerados para que Nharíne tomara la decisión, ya que este Dios les dice a los ogros que "ya no volverán a conducirse mal" (XVIII, 89) con los pótos.

\section{Referencias}

Aristóteles. (1985). Ética Nicomáquea. Ética Eudemia. Gredos: Madrid.

Bolaños-Esquivel, B. y González-Campos, G. (2010). Las miradas con que vemos. Análisis de la representación audiovisual de los pueblos indígenas de Costa Rica. San José: Vicerrectoría de Acción Social, Universidad de Costa Rica.

Carrasco-Ortiz, M. Á, y González-Calderón. (2006). “Aspectos conceptuales de la agresión: definición y modelos explicativos"en Acción Psicológica, Vol. 4, No. 2, p.7-38.

Castillo, R. (2005a). El territorio histórico maleku de Costa Rica. Revista Reflexiones, 84(1), p.71-85.

Castillo, R. (2005b). Población indígena maleku en Costa Rica. Anuario de Estudios Centroamericanos, 31,115-136.

Constenla-Umaña, A. (1992). Hagiografía y Antihagiografía en la tradición oral guatusa. Filología y Lingüística, XVIII(1), 83-124. 
Constenla-Umaña, A. (1993). Laca Majifijica. La Transformación de la Tierra. (Introducción, transcripción y traducción de Adolfo Constenla-Umaña; narración por Eustaquio Castro y Antonio Blanco). EUCR: San José.

Constenla-Umaña, A. (2011). Pláticas sobre felinos. (Narración de Eustaquio Castro; introducción, transcripción y traducción de Adolfo Constenla-Umaña). EUCR: San José.

Constenla-Umaña, A. (2014). Pláticas sobre ogros. (Narración y colaboración en la transcripción y traducción de Eustaquio Castro; introducción, transcripción y traducción de Adolfo Constenla-Umaña). EUCR: San José.

Constenla-Umaña, A. e Ibarra-Rojas, E (2014). Anotaciones etnohistóricas sobre los indígenas botos: confluencia de datos históricos, antropológicos y de la tradición oral malecu. Estudios de Lingüística Chibcha, 33, 111-164. [Este artículo contiene las 'Pláticas de bótos', en su versión malecu y española]

Marlasca-López, A. (2004). Introducción a la ética. EUNED: San José.

Rodríguez, J. M. (2010). Derechos humanos: una aproximación. FyG Editores: Guatemala.

Sánchez-Avendaño, C. (2011). Caracterización cualitativa de la situación sociolingüística del pueblo malecu. Estudios de Lingüistica Chibcha,30,63-90.

Sánchez-Avendaño, C. (2015). La cola de la iguana. El pueblo malecu ante el desplazamiento de su lengua y su cultura tradicional. EUCR: San José.

Solano-Fallas, A. (2016). "El Bien y el Mal, la Elección y la Muerte en la Cosmogonía Malecu "Laca Majifijica"”en Revista Pensamiento Actual, Vol. 16, No. 26, p.25-32. 


\section{Ogros en los malecus...}

Solano-Fallas, A; y Herrera-Valenciano, M. (2017). "Felinos en los malecus: simbolización de la muerte como aliciente moral" en Revista de Filosofía de la Universidad de Costa Rica, Vol. LVI, No. 144, Enero-Abril, p.171-178.

\section{Literatura consultada.}

Constenla-Umaña, A. (1996). Poesía tradicional indígena costarricense. (Serie antológica). EUCR: San José.

Espinoza-Romero, E; Mejía-Marín, N; y Ovares-Barquero, S. (2011). El Malecu: Una cultura en peligro de extinción. Maleku: A culture in Danger of Extintion. Revista Electrónica Educare. XV(N ${ }^{\circ}$ Extraordinario), 69-84

Fundación Coordinación de Pastoral Aborígen (FUNCOOPA)-Instituto de Estudios de las Tradiciones Sagradas de Abia Yala (IETSAY). (1999). Pueblos indígenas de Costa Rica: historia y situación actual. Tikal: San José.

Galante-Marcos, E. (directora). (sin año, A): Malecu Usirraca Marama. Libro de Leyendas Malecu. Proyecto Rio Frío. S.N. S.L.

Galante-Marcos, E. (directora). (sin año, B): Diccionario Malecu. Proyecto Rio Frío. S.N. S.L.

Guevara-Berger, M. y Chacón-Castro, R. (1992). Territorios indios en Costa Rica: orígenes, situación actual y perspectivas. García Hermanos: San José.

Ibarra-Rojas, E. (2011). Los nicaraos, los indios votos y los huetares en escenarios conflictivos en el siglo XVI. Cuadernos de Antropología, (21), 1-23. 
Junta de Educación Palenque Margarita (J.E.P. Margarita) - Instituto de Estudios de las Tradiciones Sagradas de Abia Yala (IETSAY). (2000). Narraciones malekus. Tikal: San José.

Margery-Peña, E. (2007). Estudios de mitología comparada indoamericana. Tomo II. El origen del fuego y concepciones sobre el lugar de los muertos en pueblos indoamericanos. EUCR: San José.

Mejía-Marín, N. (1994). Historias malecus. (Narración y traducción de Noemy Mejía-Marín; introducción y recopilación de Raúl Bolaños Arce, Juan de Dios Ramírez Gatgens, y Rocío Alvarado Cruz). EUNA: Heredia.

Rodríguez-Ramírez, C.A. (2015). Ética profesional docente. EUNED: San José.

Salazar-Salvatierra, R. (2006). El indígena costarricense: una visión etnográfica. The Costa Rican indigenous people: an ethnographic overview. Editorial Tecnológica de Costa Rica: Cartago.

Sánchez-Avendaño, C. (2013). Apropiación por parte de los miembros del pueblo malecu de la ortografía práctica de su lengua. Estudios de Lingüistica Chibcha, 32, 209-229.

Solano-Salazar, E: La población indígena en Costa Rica según el censo 2000. En L. Rosero Bixby (ed.). (2004). Costa Rica a la luz del censo del 2000. Centro Centroamericano de Población de la Universidad de Costa Rica: San José.

Tenorio-Alfaro, L. (1990). Reservas indígenas de Costa Rica. San José: Imprenta Nacional. .

Zavala, M. y Araya, S. (2008). Literaturas indigenas de Centroamérica (Segunda edición revisada). Heredia: EUNA. 


\section{¿Cómo citar este artículo?}

Solano Fallas, A. (Julio-diciembre, 2018). Ogros en los malecus: sobre la elección y la responsabilidad en la filosofía antigua malecu. Revista humanidades, 8(2), 1-29. doi: http://dx.doi.org/10.15517/h.v8i2.33340 